\title{
Técnicas de identificação humana em Odontologia Legal
}

\author{
Human identification techniques in Forensic Dentistry \\ Técnicas de identificación humana en Odontología Forense
}

Recebido: 22/02/2021 | Revisado: 26/02/2021 | Aceito: 04/03/2021 | Publicado: 12/03/2021

\author{
Maria Luiza Gioster-Ramos \\ ORCID: https://orcid.org/0000-0002-7002-592X \\ Universidade Estadual Paulista "Júlio de Mesquita Filho", Brasil \\ E-mail: luiza.gioster@unesp.br \\ Evelin Carine Alves Silva \\ ORCID: https://orcid.org/0000-0002-6424-5504 \\ Universidade Estadual Paulista "Júlio de Mesquita Filho", Brasil \\ E-mail: evelin.silva@unesp.br \\ Camyla Rodrigues Nascimento \\ ORCID: https://orcid.org/0000-0002-9031-6601 \\ Universidade Estadual Paulista "Júlio de Mesquita Filho", Brasil \\ E-mail: cr.nascimento@unesp.br \\ Clemente Maia da Silva Fernandes \\ ORCID: https://orcid.org/0000-0002-5401-6265 \\ Universidade Estadual Paulista "Júlio de Mesquita Filho", Brasil \\ E-mail: c.face@ terra.com.br \\ Mônica da Costa Serra \\ ORCID: https://orcid.org/0000-0001-8820-2982 \\ Universidade Estadual Paulista “Júlio de Mesquita Filho", Brasil \\ E-mail: monica.serra@unesp.br
}

\begin{abstract}
Resumo
A identificação humana é de extrema importância em questões jurídicas e sociais. Vários métodos foram desenvolvidos e aprimorados ao logo dos anos para se estabelecer a identidade individual através da odontologia forense. O objetivo do presente estudo foi analisar por meio de uma revisão da literatura, técnicas utilizadas pela Odontologia Legal na identificação humana. Para isso foram levantados artigos em sua íntegra sobre as seguintes técnicas de identificação forense utilizadas pela Odontologia Legal: exames radiográficos; estágios de mineralização do dente; seios paranasais; rugoscopia palatina; saliva; marcas de mordida e impressões labiais. Com exceção da técnica que utiliza as marcas de mordida, que serve apenas para casos de exclusão de identificação, todas as demais foram efetivas para a identificação humana, isoladamente ou associadas a outros métodos. Cada técnica tem vantagens, desvantagens, limitações em sua aplicação e indicações específicas que devem ser analisadas de acordo com os casos individualmente e materiais disponíveis para comparação de registros ante mortem e post mortem. Neste contexto, é essencial que o profissional da Odontologia que atua na clínica odontológica, preencha e guarde adequadamente os prontuários dos seus pacientes. Nesta era digital, a guarda de exames por imagem, sejam radiológicos (como radiografias e tomografias computadorizadas) ou não (como fotografias e escaneamentos 3D intraorais) é simples, prática, e de fundamental importância forense.
\end{abstract}

Palavras-chave: Antropologia forense; Odontologia legal; Identificação de vítimas; Ciências forenses.

\begin{abstract}
Human identification is extremely important in legal and social questions. Various methods have been developed and improved over the years to establish individual identity through forensic dentistry. The aim of this study was to analyze, through a literature review, techniques used by Forensic Dentistry in human identification. For that purpose, full articles were collected on the following forensic identification techniques used by Forensic Dentistry: radiographic exams; stages of tooth mineralization; paranasal sinuses; palatal rugoscopy; saliva; bite marks and lip impressions. With the exception of the technique that uses the bite marks that are only used for cases of exclusion of identification, all the others were effective for human identification, either alone or associated with other methods. Each technique has advantages, disadvantages, limitations in its application and specific indications that must be analyzed according to the individual cases and available materials to compare ante mortem and post mortem records. In this context, it is essential that the dental professionals, who works in the dental clinic, properly fill out and keep the records of their patients. In this digital age, keeping imaging tests, whether radiological (such as radiographs and CT scans) or not (such as photographs and intraoral 3D scans) is simple, practical, and of fundamental forensic importance.
\end{abstract}

Keywords: Forensic anthropology; Forensic dentistry; Victims identification; Forensic sciences. 


\begin{abstract}
Resumen
La identificación humana es extremadamente importante en asuntos legales y sociales. Se han desarrollado y mejorado varios métodos a lo largo de los años para establecer la identidad individual a través de la Odontología Forense. El objetivo de este estudio fue analizar técnicas utilizadas por la Odontología Legal en la identificación humana a través de una revisión de la literatura. Para ello, se recopilaron artículos sobre las técnicas de identificación forense utilizadas en la Odontología Legal tales como: exámenes radiográficos, etapas de mineralización de los dientes, senos paranasales, rugoscopía palatina, saliva, marcas de mordidas e impresiones labiales. A excepción de la técnica que utiliza las marcas de mordida, que sólo se utilizan para casos de exclusión de identificación, todas las demás resultaron eficaces para la identificación humana, ya sea solas o asociadas a otros métodos. Cada técnica tiene ventajas, desventajas y limitaciones en su aplicación con indicaciones específicas que deben analizarse según los casos individuales y los materiales disponibles para comparar los registros ante mortem y post mortem. En este contexto, es fundamental que el profesional de la odontología que trabaja en la clínica dental, complete y lleve debidamente los registros de sus pacientes. En esta era digital, guardar las pruebas de imágenes, ya sean radiológicas (como radiografías y tomografías computarizadas) o no (como fotografías y escâneres 3D intraorales) es simple, práctico y de fundamental importancia forense.
\end{abstract}

Palabras clave: Antropología forense; Odontología forense; Identificación de víctimas; Ciencias forenses.

\title{
1. Introdução
}

A identificação humana é de fundamental importância para sociedade, seja pelas considerações humanitárias ou por sua aplicação em processos cíveis e criminais (Choi et al., 2018). Vários métodos foram desenvolvidos para se estabelecer a identidade individual; para reconhecimento são amiúde considerados o reconhecimento visual de peças de vestuário e itens pessoais. (Tatlisumak et al., 2011). Métodos de identificação considerados primários, sobretudo em desastres de massa, são datiloscopia, perfil de DNA e Odontologia Forense (Interpol, 2018). O cirurgião-dentista tem papel primordial no sucesso da correta identificação, ao fazer um correto arquivamento de prontuários, radiografias, modelos em gesso dos pacientes e anotações sobre cada caso, pois estas podem servir para a identificação positiva de vítimas (Andrade et al., 2021).

Odontologia Legal, ou Odontologia Forense, é um ramo da Odontologia que envolve a aplicação da ciência odontológica para a identificação de restos humanos desconhecidos, através da comparação de dados ante mortem com dados post mortem, sendo o principal método utilizado para a identificação de vítimas em casos de desastre em massa, por desempenhar um papel básico na identificação de indivíduos que não podem ser identificados por métodos de rotina (Choi et al., 2018).

Não se pode deixar de destacar também a possibilidade de necessidade de identificação de indivíduos vivos. Da mesma forma, é preciso um primeiro registro, sabida e comprovadamente de uma determinada pessoa, para ser comparado com o segundo registro, obtido no momento da aplicação do método de identificação.

A Odontologia Forense é imprescindível nos processos que envolvem identificação humana, realizando, por exemplo, estimativas de sexo, idade e estatura, diagnóstico de manchas de sangue e saliva, e atuando na definição da causa e do tempo de morte (Oliveira et al., 1998).

A atuação do cirurgião-dentista na seara da Odontologia Legal está assegurada pela legislação federal, por meio da Lei $\mathrm{n}^{\circ}$ 5.081, de 24 de agosto de 1966 (Brasil, 1966), que regulamenta o exercício da Odontologia no Brasil. E pelo Conselho Federal de Odontologia, por meio da Resolução nº 63, de 08 de abril de 2005 (Brasil, 2005) - Consolidação das Normas para Procedimentos nos Conselhos de Odontologia, que define o campo de atuação do especialista em Odontologia Legal como, entre outros, identificação humana, tanatologia forense, infortunística, traumatologia, balística forense, e imaginologia.

Segundo Moreira e Freitas (1999), a identidade corresponde ao "conjunto de caracteres que permitem distinguir uma pessoa das demais, individualizando-a física e juridicamente", enquanto identificação é o "processo técnico científico pelo qual se determina a identidade de uma pessoa ou de uma coisa, ou um conjunto de diligências cuja finalidade é levantar uma identidade: a identificação". Para que um método de identificação seja considerado ideal, deve preencher os requisitos da unicidade, imutabilidade, praticidade, classificabilidade e perenidade. 
Diferentes métodos para a identificação humana são utilizados pelos odontolegistas, sendo alguns deles: exame de registros odontológicos, que confronta a documentação odontológica prévia de um suspeito com o indivíduo a ser identificado, por comparação de exames radiológicos (Page et al., 2018), tomográficos e clínicos, análise de modelos de gesso, dos dados dos tratamentos realizados com aspectos da cavidade bucal da vítima (Afify et al., 2014); marcas de mordida (Freeman, Senn \& Arendt, 2005); análise dos seios da face (Bangi et al., 2017) ; análise de saliva (Tsai et al., 2018); análise de rugas palatinas (Santos, Fernandes \& Serra, 2011; Poojya et al., 2015); estimativa de idade por estágios de mineralização dentária (Bleka et al., 2019),impressões labiais (Sharma, Saxena \& Rathod, 2009; Herrera, Fernandes \& Serra, 2013; Herrera, Fernandes \& Serra, 2018) e marcas de orelha (Silva, Fernandes \& Serra, 2012).

O presente estudo teve como objetivo analisar, através de uma revisão de literatura, algumas técnicas utilizadas pela Odontologia Forense em identificação humana. Não se trata de uma lista de numerus clausus, ou seja, não é uma relação fechada, não englobando a totalidade das possibilidades de técnicas utilizadas pela Odontologia Legal.

\section{Metodologia}

Foi realizada uma busca abrangente nas bases de dados da literatura (PubMed, Medline, Scopus, Web of Science e Google Scholar) utilizando uma combinação das palavras-chave "bite mark", "forensic dentistry", "mineralization age estimation, "palatal rugae", "palatoscopy", "frontal sinus", "human identification", "maxillary sinuses", "paranasal sinuses", "diagnostic imaging"," cheiloscopy" e "lip prints". Não foi determinado período, sendo selecionados artigos completos que discorriam sobre as técnicas de identificação humana aplicadas na Odontologia Forense. Como critérios de inclusão foram utilizados artigos científicos publicados em revistas nacionais e internacionais sobre o tema, disponibilizados na íntegra.

\section{Resultados e Discussão}

\section{Exames imaginológicos}

A identificação humana por meio da Odontologia Forense depende da verificação de características concordantes comuns, realizada por meio de comparação de registros ante mortem e post mortem, no caso de identificação de cadáveres e ossadas.

A radiologia odontológica tem desempenhado um papel fundamental na identificação das vítimas em muitos casos. Em 1973, a identificação de 73\% das 35 vítimas queimadas no Hotel Hafnia, na Dinamarca, foi realizada por oito cirurgiõesdentistas através da utilização de radiografias dentárias (Petersen, 1975). A identificação das vítimas americanas da Operação Tempestade no Deserto foi amplamente dependente da radiologia odontológica forense, onde de 251 vítimas, 244 foram identificadas com base na disponibilidade de registros odontológicos, incluindo imagens radiográficas panorâmicas (Kessler \& Pemble, 1993).

Características anatômicas, como tamanho e forma das coroas, anatomia pulpar, e posição e forma da crista do osso alveolar, podem ser muito úteis. No entanto, mais utilizadas ainda são as mudanças originadas por cáries e as restaurações. O tratamento dentário resulta em características únicas e individuais que, na maioria das vezes, são bem visíveis nas radiografias comuns. Assim, a técnica de identificação consiste essencialmente numa comparação entre radiografias tiradas em vida (ante mortem), arquivadas nos consultórios dentários, com as obtidas após a morte (post mortem) (Gruber \& Kameyama, 2001).

Um estudo realizado por Page et al. (2018) demonstrou que apenas pessoas com qualificações e experiência odontológica devem ser consideradas para realizar a tarefa de comparação de radiografias. Os resultados encontrados pelos autores demonstraram que, ao declarar uma correspondência em um modelo de escolha binária, é provável que seja uma correspondência verdadeira 89,3\% das vezes. Por outro lado, uma não correspondência declarada provavelmente será uma não correspondência verdadeira $85,6 \%$ das vezes. 
O padrão de restauração de amálgama foi investigado por Philips, que descobriu que os padrões de restauração de amálgama no primeiro molar eram relativamente comuns e, portanto, tinham uma medida baixa de singularidade. No entanto, se o padrão da restauração de amálgama no primeiro molar fosse combinado com os padrões em um ou mais outros dentes, então a singularidade aumentou acentuadamente e melhorou a probabilidade de identificação da pessoa (Kessler \& Pemble, 1993).

As radiografias endodônticas pós-operatórias fornecem uma rica fonte de informações que podem ser características únicas de identificação de um dente e indivíduo em particular. Isso se deve à rara frequência de variação entre a aparência radiográfica de uma restauração endodôntica em comparação com a de uma restauração intracoronal (Forrest \& Wu, 2018). Bonavilla et al. (2008) realizaram um estudo para avaliar quais materiais de obturação endodôntica eram capazes de suportar altas temperaturas, como aquelas às quais uma vítima incinerada teria sido exposta. Essas informações foram usadas para compilar um banco de dados sobre o uso de materiais obturadores de canais radiculares como auxílio na identificação forense. Khalid et al. (2016) demonstraram que as imagens radiográficas dos canais obturados de dentes unirradiculares apresentaram características morfológicas altamente específicas, que poderiam atuar como um auxílio potencial para fins de identificação. O potencial discriminatório da morfologia única do canal obturado de um dente unirradicular pode ser usado para a tomada de decisão baseada em evidências em Odontologia Legal.

Serra, Herrera \& Fernandes (2012) apresentaram um relato de caso de identificação em que foi realizada comparação da radiografia final de um tratamento endodôntico com a radiografia post mortem do mesmo dente, além da constatação de outras coincidências. Os autores destacam a necessidade de confecção e guarda corretas do prontuário odontológico, que pode ser eventualmente requisitado para fins de identificação.

A radiografia panorâmica também tem se mostrado uma ferramenta importante para a estimativa de diferentes parâmetros morfológicos da mandíbula. Essas radiografias têm reprodutibilidade aceitável para a mensuração de variáveis verticais e angulares para comparação de grupos, desde que o posicionamento da cabeça seja padrão e mantido constante. Além disso, a sobreposição de marcos anatômicos como no cefalograma lateral e o aumento da exposição à radiação como na tomografia computadorizada e na tomografia computadorizada de feixe cônico são relativamente menores nesta modalidade. As alterações de remodelação da mandíbula em relação às faixas etárias e estado dentário são mínimas (Chidambaram, 2016).

Avanços recentes na tecnologia digital médica e odontológica influenciaram o desenvolvimento e a aplicação da Odontologia Forense. Em particular, a digitalização de exames de imagem odontológica tem facilitado a análise de imagens, em particular por meio do uso de computadores que permitem a medição de várias estruturas anatômicas. Também facilitaram a manutenção de informações em bancos de dados digitais e a movimentação de imagens (Matsuda et al., 2020).

A tomografia computadorizada 3D Multislice e de feixe cônico, em Odontologia Forense, demonstrou ser útil em vários casos de necessidade de identificação, em acidentes. O estudo de Eliášová \& Dostálová (2017) demonstrou que a radiologia digital e a tomografia computadorizada têm se mostrado importantes tanto nas práticas comuns de criminalística quanto em vários incidentes fatais, e que a imagem da tomografia computadorizada de feixe cônico (TCFC) oferece menos artefatos de imagem e tempos de reconstrução de imagem baixos.

Krishnan et al. (2019) ressaltam, porém, que existem possibilidades de ocorrência de erros graves na radiografia odontológica digital devido à função de rotação e inversão das imagens pelo software, de forma que as informações para identificação dentária individual devem ser usadas em combinação com outras evidências ou achados circunstanciais.

\section{Estágios de mineralização dentária}

A estimativa de idade de uma vítima ou de um suspeito de delito é de fundamental importância para identificação, além de delinear os passos seguintes de uma investigação. Umas das formas de determinar a idade cronológica do indivíduo é 
por meio dos estágios de mineralização dos dentes. Em se tratando de identificação de restos mortais, os dentes, diferentemente das outras estruturas do corpo, apresentam maior resistência às adversidades ambientais (Oliveira et al., 2012).

A dentição humana permanente se inicia por volta dos 6 anos e finaliza acerca dos 18 anos - que na maioria dos países representa a maioridade penal. Por meio de radiografias foram estabelecidos diversos métodos capazes de alcançar valores bem próximos da idade real do indivíduo, analisando o estágio de mineralização que o dente se encontra (Khorate, Dinkar \& Ahme, 2014).

Por serem os últimos dentes a erupcionar, os terceiros molares são os dentes mais utilizados para aplicação da técnica em adultos jovens, apesar da agenesia desses dentes ser a mais frequente, por volta dos 18 anos eles ainda não concluíram o desenvolvimento (Lopez et al., 2013).

Vários autores concentraram-se em relacionar os estágios de mineralização dos dentes com a idade dos sujeitos analisados, desenvolvendo fórmulas utilizadas para a análise das radiografias com o objetivo de chegar o mais próximo da idade real (Urzel \& Bruzek, 2013). Dentre os métodos mais utilizados estão de Nolla (1952), Demirjian et al. (1973), Willems et al. (2001), e Cameriere, Ferrante e Cingolani (2006). O grande impedimento da utilização dessa técnica para identificação humana está no fato de que a ancestralidade dos indivíduos influenciará no seu desenvolvimento como um todo, afetando dessa maneira, a fase para mineralização dos dentes (Flood et al., 2011).

Assim, valores encontrados em jovens brasileiros, provavelmente não se aplicarão para jovens suíços, por exemplo. Essa dificuldade é consensual entre os autores e claramente impede o desenvolvimento de um método único, levando à necessidade de cada população realizar suas próprias pesquisas e obter um método reprodutível e confiável (Jayaraman et al., 2012; Gaêta-Araujo et al., 2021). Além disso, em países que possuem grande miscigenação, como o Brasil, há uma dificuldade ainda maior para estabelecer um padrão para os estágios de mineralização.

\section{Seios paranasais}

Os seios paranasais são cavidades pneumáticas que existem em alguns ossos, possuem grande diversidade em sua morfologia, e a possibilidade de duas pessoas terem a mesma morfologia é remota (Nambiar, Naidu \& Subramaniam 1999), o que permite a sua utilização para identificação humana. Pode-se utilizar radiografias intraorais e panorâmicas e tomografias computadorizadas. Estas possuem melhor resolução, sem sobreposição de elementos e a possibilidade de mensuração mais precisa, sendo assim a técnica de escolha para análise dos seios da face (Uthman et al., 2010).

Foram encontrados estudos que utilizavam os seios esfenoides (Cappella et al., 2019), maxilar (Bangi et al., 2017; Prabhat et al., 2016), e frontal (Yoshino et al., 1986; Christensen, 2005; Cameriere et al., 2008; Uthman et al., 2010; Besana \& Rogers, 2010; David \& Saxena 2010; Patil et al., 2012; Kim et al., 2013; Goyal et al., 2013; Hamed et al., 2014; Nikam et al., 2015; Beaini et al., 2015; Michel et al., 2015; Akhlaghi et al., 2016; Verma et al., 2017; Choi et al., 2018; Gibelli et al., 2019). O seio frontal é uma parte resistente do crânio de fácil recuperação para utilização em investigações forenses (Akhlaghi et al., 2016).

Para identificação por comparação dos seios da face, diversas técnicas foram citadas, dentre elas a análise elíptica de Fourier (EFA), uma técnica morfométrica geométrica que impõe um conjunto de pontos coordenados para uma curva fechada, no intuito de quantificar a borda superior dos seios frontais através das distâncias euclidianas (Christensen, 2005). O método de Yoshino divide os padrões do seio frontal acima de 20.000 combinações possíveis, combinando os números das classes em cada item de classificação (Yoshino et al., 1986), e o de Cameriere é um método de classificação do seio frontal (Cameriere et al., 2008).

A seguir citamos alguns métodos utilizados para identificação, utilizando o seio frontal. Através do número de septos parciais no seio frontal direito e a altura máxima e largura por radiografias cefalométricas (Patil et al., 2012; Akhlaghi et al., 
2016; Verma et al., 2017). Por combinações discretas de traços e correspondência de padrões de sobreposição (Besana \& Rogers, 2010). Através do software ITK-SNAP e sobreposição de modelos 3D correspondentes. Da aquisição de imagens 3D em tomografia do volume da cavidade e medidas da área, perímetro, retângulo delimitador, ajuste de elipse, circularidade, proporção, arredondamento, solidez e diâmetro defere (Choi et al., 2018). Das medidas de volume total do seio, algumas características não métricas, e o índice de assimetria bilateral (Kim et al., 2013). Utilização do visualizador de código aberto DICOM InVesallius ${ }^{\circledR}$ para renderização, Mesh Lab®, para posicionamento dos modelos e CloudCompare para comparação volumétrica dos seios (Beaini et al., 2015). Pelo volume do seio frontal calculado em mm3 após tomografia (Michel et al., 2015). Pela medida e comparação das dimensões para os seios direito e esquerdo (transversal, altura e comprimento ânteroposterior), das seções axiais e coronais (espessura de $4 \mathrm{mms}$ ) em tomografia computadorizada (Hamed et al., 2014). Segmentação no software de código aberto ITK-SNAP e sobreposição dos modelos 3D (Gibelli et al., 2019). Utilização de Imagens de microtomografia para medir as dimensões médio-lateral supero-inferior e ântero-posterior e o volume dos seios maxilares (Prabhat et al., 2016; Bangi et al., 2017). Nas radiografias cefalométricas anteriores posteriores o seio frontal foi classificado de acordo com simetria, assimetria dominante direita ou esquerda e unilateral e aplasia bilateral (David \& Saxena, 2010). Pela presença ou ausência de seio frontal, de septo e escalonamento (Uthman et al., 2010).

E apesar das diferentes formas de se medir e comparar os seios paranasais, a maioria dos artigos concorda que os seios da face, pela sua singularidade entre pessoas, servem para identificação humana, sendo capazes de identificar com precisão os indivíduos, apresentando de 72,5\% a 100\% de acurácia (Yoshino et al., 1986; Christensen, 2005; Cameriere et al., 2008; Besana \& Rogers, 2010; David \& Saxena, 2010; Patil et al., 2012; Kim et al., 2013; Goyal et al., 2013; Beaini et al., 2015; Nikam et al., 2015; Prabhat et al., 2016; Verma et al., 2017; Cappella et al., 2019; Gibelli et al., 2019 ) e para dimorfismo sexual ( Hamed et al., 2014; Michel et al., 2015; Bangi et al., 2017; Choi et al., 2018), e apenas um não validou o método como confiável (Akhlaghi, et al., 2016) .

\section{Rugoscopia palatina}

As rugas palatinas são elevações assimétricas e irregulares da mucosa bucal situadas no terço anterior do palato (Patil, Patil \& Acharya, 2008). Devido às suas características permanentes e únicas para cada indivíduo (Adisa, Kolude \& Ogunrinde, 2014), por estarem presentes em todas as pessoas e serem resistentes a alterações como o envelhecimento e traumas (Kommalapati et al., 2017), a rugoscopia palatina é indicada para identificação humana, através da análise das rugas palatinas. A utilização de padrões de rugas palatinas para identificação humana foi sugerida por Allen em 1889.

Para se proceder à análise da rugoscopia é necessária a comparação de formas, números e classificação das rugas palatinas (Santos, Fernandes \& Serra, 2011). A inspeção intraoral é o método mais usado por ser fácil e econômico, pode se avaliar também por fotografias, fazendo impressões em modelos de gesso, digitalização por scanner a laser 3D de modelos por estereoscopia, que obtêm uma imagem tridimensional da anatomia das rugas palatinas. Para a sobreposição de várias fotografias digitais para se comparar os padrões de rugas palatinas, usa-se também variados computadores e programas (Sunil et al., 2014). A tecnologia de digitalização tridimensional (3D) facilita a correspondência computadorizada dos padrões de rugas palatinas de forma segura e comprovada, comparável ao padrão ouro atual que avalia impressões digitais (Taneva, Kusnoto \& Evans, 2015)

Com base nos estudos levantados, verificamos a presença de dúvida relativa à interferência do uso de aparelhos ortodônticos no padrão das rugas, mas apesar disso a maioria dos estudos comprovou que a forma de rugas permaneceu consistente (Pateria \& Thakkar, 2011; Taneva, Kusnoto \& Evans, 2015; Ali, Shaikh \& Fida, 2016). Um estudo apenas afirmou que o tratamento ortodôntico induz alterações morfométricas que podem dificultar a identificação humana baseada em rugas palatinas, mas não impedir (Mustafa, Allouh \& Alshehab, 2015). Quando avaliados os métodos, a inspeção visual se mostrou 
um desafio, pois precisa de uma calibração prévia (Adisa, Kolude, \& Ogunrinde, 2014). A utilização de fotografias com posterior processamento em softwares permite uma classificação das rugas palatinas (Manganotti et al., 2021). A sobreposição 2D e 3D foi capaz de distinguir corretamente sem erros o grupo de correspondências (Santos, Fernandes \& Serra, 2011; Taneva, Evans \& Viana, 2017; Gibelli et al., 2018).

Para estimativa de sexo, as rugas palatinas se mostraram adequadas, segundo alguns autores (Gadicherla, Saini \& Bhaskar, 2017; Malekzadeh et al., 2018; Gaikwad et al., 2019); para identificação individual as rugas palatinas foram consideradas em sua maioria como um parâmetro de identificação forense ideal, com até $100 \%$ de correspondência (Santos, Fernandes \& Serra, 2011; Shukla et al., 2011; Jain \& Chowdhary, 2014; Poojya et al., 2015; Ali, Shaikh \& Fida, 2016; Taneva, Evans \& Viana, 2017; Gibelli et al., 2018; Castro-Espicalsky et al., 2020). Podem também ser consideradas como uma técnica auxiliar ou principal no processo de identificação humana (Manganotti et al., 2021).

\section{Queiloscopia}

A queiloscopia é um método de identificação humana, onde são analisados, os sulcos labiais, suas disposições, as comissuras labiais e a espessura labial do indivíduo (Herrera, Fernandes \& Serra, 2013; Herrera, Fernandes \& Serra, 2018; Caldas, Magalhaes \& Afonso, 2007). Ayuba et al. (2019) afirmam que o padrão de impressão labial e a espessura dos lábios variam entre as populações; essa variação pode ajudar em investigações criminais e forenses. Geralmente as impressões labiais são classificadas de acordo com sistema de classificação de Suzuki e Tsuchihashi (1970) e posteriormente por People v. Davis (Fonseca \& Cantín, 2014). A queiloscopia como técnica de identificação humana, respeita os requisitos necessários para serem intituladas como tal, sendo perenes, imutáveis, de fácil obtenção e classificáveis (Santos, 1946).

Devido às impressões labiais serem únicas, e análogas às impressões digitais, podem ser usadas para identificação pessoal de um criminoso no tribunal. Quando não existem outras evidências disponíveis, pode se encontrar impressões labiais latentes em vidros, copos, papéis, roupas, pontas de cigarro, vidraças, etc. (Kaul et al., 2015). Frequentemente, a qualidade da impressão labial é muito ruim; ou muito borrada, o que torna impossível a identificação queiloscópica. Além de algumas delas serem impressões latentes, que só podem ser reveladas com pós de impressão digital, como pó preto, pó cinza e até mesmo tinturas lisocromáticas (Segui et al., 2000), mas das quais se pode extrair o DNA seguida pela tipagem dos loci STR, estabelecendo as impressões dos lábios como evidência biológica (Sharma et al., 2016).

De acordo com estudos levantados, observamos que padrões adequados e controle de viés e erro de observação devem ser implementados para evitar erros e identificações incorretas. As evidências de impressões labiais são amiúde subdiagnosticadas, pois muitos Peritos não são suficientemente treinados para obtenção das mesmas (Fonseca et al., 2019). Porém, quando avaliadas corretamente, pode-se obter eficácia de 92,73\% e uma taxa de identificação de 100\% (Wrobel et al., 2018). Quando comparada a relação da queiloscopia com sua representação entre descendentes, o padrão sulcular labial se fez menos reprodutível entre mães e filhas do que a espessura do lábio e a posição/forma da comissura labial (Silva et al., 2020). As impressões labiais são indicadas como um auxílio confiável, fácil e barato para a identificação humana no campo da Odontologia Forense, desde que se empreguem técnicas que combinem análise analógica, digital e classificação (Ulery et al., 2011; Kaul et al., 2015; Sharma et al., 2016; Wrobel et al., 2018; Fonseca et al., 2019).

A queiloscopia é uma técnica considerada confiável na identificação humana, como também na determinação do sexo de um indivíduo (Thermadam, Chatra \& Ahsan, 2020). Mas para a sua utilização, se é exigida cautela na interpretação de dados para a identificação humana, mais estudos na área devem ser realizados para que se tenham níveis de evidência mais confiáveis e assim assegurar a aplicação prática do método queiloscópico (Silva et al., 2020). 


\section{Saliva}

A saliva é o meio de escolha entre todos os fluidos corporais em investigações forenses e criminais devido à facilidade de disponibilidade, e métodos de coleta não invasivos e econômicos. Os avanços na tecnologia do DNA sem dúvida melhoraram o isolamento do DNA humano em manchas de saliva secas obtidas a partir de marcas de mordidas e impressões labiais, e o tornaram mais preciso. A saliva também pode ser utilizada para perfis biológicos, para determinar idade, sexo e características pessoais de indivíduos para identificação reconstrutiva (Poojya et al., 2015).

A saliva é um espelho da saúde bucal e também um reservatório de analitos de fontes sistêmicas que chegam à cavidade oral por diversas vias. A composição molecular da saliva reflete os níveis de fluido do tecido de moléculas terapêuticas, hormonais, imunológicas ou toxicológicas. Além disso, marcadores de doenças podem estar presentes. Consequentemente, esses fluidos fornecem fontes para avaliação e monitoramento de estados de saúde e doenças sistêmicas, exposição a toxinas ambientais e relacionadas ao trabalho e o uso de drogas abusivas ou terapêuticas (Kapoor \& Chowdhry, 2018).

Embora seja facilmente acessível de maneira não invasiva, a saliva tem a vantagem de que o background do material normal (células, DNA, RNA e proteínas) e substâncias inibidoras é muito menor e menos complexo do que no sangue. Em Ciências Forenses, um teste de base molecular permite a identificação de manchas de fluido corporal, demonstrando a utilidade e validade do teste de mRNA salivar (Poojya et al., 2015; Kapoor \& Chowdhry, 2018).

Khare et al. (2007) constataram que a saliva pode ser depositada na pele humana por meio de mordidas, chupadas, lambidas, beijos, etc. É encontrada em vítimas de vários crimes violentos e potencialmente recuperada e digitada a partir de marcas de mordidas, pontas de cigarro, selos postais, envelopes, comestíveis e outros objetos.

De modo consensual entre os autores analisados, a saliva é descrita como uma fonte muito boa de DNA humano, pois o número médio de células epiteliais por $1 \mathrm{~mL}$ de saliva é cerca de 4,3 × 105 . Além disso, a renovação das células epiteliais é bastante extensa na boca, pois a camada superficial das células epiteliais é substituída, em média, a cada 2,7 horas, sugerindo que é provável que haja DNA genômico intacto nas amostras de saliva (Below et al., 2018; Muruganandhan \& Sivakumar, 2011).

O uso da saliva como fonte de DNA permite certas vantagens técnicas em relação ao uso do sangue. A coleta é mais fácil e indolor, principalmente considerando que pode ser realizada em bebês, crianças e idosos, e não tem as implicações religiosas do uso do sangue. Muruganandhan e Sivakumar (2011) constatam em seu estudo que a saliva pode ser uma boa fonte de DNA e pode ser usada com sucesso para fins forenses.

\section{Marcas de mordida}

Marcas de mordida são consideradas um dos meios de identificação controversos e de maior complexidade para utilização dentro das técnicas de Odontologia Forense (Van der Velden, Spiessens \& Willems, 2006). São apontadas como únicas para cada indivíduo e normalmente são caracterizadas por duas impressões em forma de "U”, representando os arcos superior e inferior, acompanhadas, na maioria das vezes, por uma equimose entre os arcos (Pretty \& Hall, 2002).

As estratégias mais comuns para determinação das marcas de mordida são por meio de comparação da morfologia da dentição com características e posicionamento análogos encontrados em fotografias em tamanho real das injúrias por meio de sobreposição transparente ou com auxílio de programas de computador. Outro meio de determinação é com a utilização dessas impressões verificadas diretamente com o modelo de estudo do suspeito e as imagens obtidas das marcas de mordida (Santoro et al., 2011).

O motivo pelo qual a técnica não é tão confiável e pode levar a muitos questionamentos diante de um tribunal, por exemplo, é o fato de que o método não fornece valores quantitativos para mensuração das injúrias, e nos casos em que o 
suspeito não é definido, o Perito não pode definir que as marcas encontradas são de um indivíduo em particular. O método pode apenas elucidar se há ou não probabilidade de que a marca de mordida pertença a algum dos suspeitos. Ou seja, quando somente essa evidência é apresentada ela não será suficiente para determinar a culpado (Tuceryan et al., 2011).

Outra razão para o descrédito da técnica é devido ao fato de as marcas de mordida normalmente estarem associadas a crimes brutais como estupro, agressões e abusos infantis. No momento em que essas impressões são realizadas, podem ocorrer distorções de acordo com as reações que a vítima pode ter, considerada essa, distorção primária. Já no momento em que as lesões são registradas pelos Peritos poderá ocorrer uma distorção secundária, pois dificilmente será replicada a posição exata em que o criminoso deixou a mordida e o posicionamento correto do corpo da vítima. Dessa maneira, a identificação do suspeito torna-se difícil, pois as marcas deixadas dificilmente se encaixarão com um perfil, diante de tantas alterações e, além disso, as características da pele da vítima também representam um meio que pode modificar as marcações (Sheasby \& MacDonald, 2001).

Apesar disso, as marcas de mordida podem ser úteis para descartar um suspeito ou em casos em que a amostra de prováveis culpados representa uma população fechada, ou seja, todos os suspeitos do caso são conhecidos - assim a análise das marcas de mordida pode ser de grande ajuda (Raina, Kulkarni \& Shah, 2019). Em um relato de caso, Weeratna (2014), mostrou que por meio da moldagem dos arcos dentários de familiares foi possível identificar qual era o responsável pelas injúrias causadas em uma criança de 3 anos.

Dessa maneira, é bem esclarecido que a técnica tem suas limitações, mas quando bem aplicada fornecerá as respostas adequadas ao odontolegista. Apesar disso, é observado que o método tem sido cada vez mais substituído por procedimentos mais assertivos e atualmente, a presença de uma marca de mordida na vítima representa mais uma possível fonte de DNA do culpado do que uma técnica de identificação em si (Roh et al., 2018).

\section{Considerações Finais}

A Odontologia Legal, ou Odontologia Forense, considerada como método primário de identificação humana, possui diversas técnicas que podem ser empregadas em processos de identificação humana. É importante ressaltar que, como todo método de identificação (e não de reconhecimento), é preciso um primeiro registro (neste caso, prontuários odontológicos, exames por imagem, modelos em gesso, etc.), para que possa ser comparado com o segundo registro. Neste contexto, destacase a fundamental importância da confecção e guarda dos prontuários odontológicos pelos cirurgiões-dentistas.

Os exames imaginológicos são fontes de dados ante mortem (ou primeiro registro) facilmente armazenados e existentes em prontuários odontológicos, que guardam informações de tratamentos prévios endodônticos, restauradores, cirurgias e implantes, além de possuírem imagens dos seios da face, que podem ser usadas para identificação pela singularidade dos seios em cada indivíduo. A rugoscopia palatina e a queloscopia são indicadas para identificação individual, apesar de existirem poucos estudos que padronizem seu uso e capacitem os profissionais para fazer uso das mesmas. Não obstante, atualmente, com um maior emprego de escaneamentos intraorais (além das fotografias digitais), é possível o armazenamento simples, digital, de informações referentes às rugas palatinas (o que, até há pouco tempo, somente era possível por meio da guarda de modelos em gesso do arco superior, que abrande o palato).

As marcas de mordida atualmente são mais utilizadas como excludentes de identificação quando existe dúvida entre mais de um possível delinquente, mas é possível utilizá-las como fonte de DNA através da saliva, que é mais fácil e indolor do que o sangue para obtenção de amostras de DNA, sendo um meio seguro para fins forenses.

Estágios de mineralização dentária, analisados para estimar-se a idade de um sujeito, são influenciados pela ancestralidade dos indivíduos; apesar de ser um método seguro e replicável, para cada população deve ser utilizada uma tabela de referência confeccionada com dados específicos. Para a população brasileira, são necessários estudos atuais sobre o tema. 
Diversas técnicas de identificação humana abrangem a área de Odontologia Forense, demonstrando a importância da atuação de um profissional odontolegista nas Ciências Forenses. Cada técnica apresenta vantagens, desvantagens e indicações específicas que devem ser analisadas individualmente e escolhidas para serem aplicadas isoladamente ou somadas a outras técnicas, em casos de identificação humana.

A Odontologia Forense, ao lado da datiloscopia e da análise de DNA, é método de identificação primário. Nem sempre é possível utilizar impressões digitais, que podem não estar presentes (como em carbonizados ou ossadas). A análise de DNA demanda tempo, tem custo elevado e muitas vezes inexiste um primeiro registro disponível, para que seja realizada a comparação. Os métodos empregados pela Odontologia Forense são, via de regra, práticos, simples, de baixo custo e confiáveis, tornando-se, em muitas situações, a alternativa que responde à Justiça e à sociedade o questionamento sobre a identidade do de cujus. Em desastres de massa, informações odontológicas são também imprescindíveis para a identificação das vítimas. Neste contexto, é essencial que o profissional da Odontologia que atua na clínica odontológica, preencha e guarde adequadamente os prontuários dos seus pacientes. Nesta era digital, a guarda de exames por imagem, sejam radiológicos (como radiografias e tomografias computadorizadas) ou não (como fotografias e escaneamentos 3D intraorais) é simples, prática, e de fundamental importância forense.

\section{Agradecimentos}

O presente trabalho foi realizado com apoio da Coordenação de Aperfeiçoamento de Pessoal de Nível Superior Brasil (CAPES) - Código de Financiamento 001.

\section{Referências}

Adisa, A. O., Kolude, B., \& Ogunrinde, T. J. (2014). Palatal rugae as a tool for human identification. Niger J Clin Pract, 17(5): $641-643$.

Ali, B., Shaikh, A. \& Fida, M. (2016). Stability of palatal rugae as a forensic marker in orthodontically treated cases. J Forensic Sci, $61(5)$ : $1351-1355$.

Allen H. (1889). The palatal rugae in man. Dent. Cosmos, 31: 66-80.

Akhlaghi, M., Bakhtavar, K., Moarefdoost, J., Kamali, A. \& Rafeifar, S. (2016). Frontal sinus parameters in computed tomography and sex determination. Legal Med, 19: 22-27.

Afify, M. M., Zayet, M. K., Mahmoud, N. F. \& Ragab, A. R. (2014). Age estimation from pulp/tooth area ratio in three mandibular teeth by panoramic radiographs: Study of an Egyptian sample. J Forensics Res, 5(3): 1.

Andrade, A. M. C., Gomes, J. A., Oliveira, L. K. B. F., Santos, L. R. S., Silva, S. R. C., Moura, V. S. \& Romão, D. A. (2021). Odontologia legal-o papel do Odontolegista na identificação de cadáveres: uma revisão integrativa. Res Soc Dev, 10(2): e29210212465.

Ayuba, J. T., Echoru, I., Ssempijja, F., Ann, M. L., Edgar, F. \& Buhari, M. (2019). Sexual dimorphism in the lip print pattern and size among Ugandan, Kenyan and Somali population. FSI: Reports, 1: 100012.

Bangi, B. B., Ginjupally, U., Nadendla, L. K. \& Vadla, B. (2017). 3D evaluation of maxillary sinus using computed tomography: A sexual dimorphic study. Int J Dent, 2017: 9017078.

Beaini, T. L., Duailibi-Neto, E. F., Chilvarquer, I. \& Melani, R. F. (2015). Human identification through frontal sinus 3D superimposition: Pilot study with Cone Beam Computer Tomography. J Forensic Leg Med, 36: 63-69.

Below, H., Baguhl, R., Geßner, W., Kramer, A., Below, E., Kahlert, H. \& Welk, A. (2018). Specific and robust ion chromatographic determination of hypothiocyanite in saliva samples. Anal. Biochem Chemistry, 410(11): 2739-2749.

Besana, J. L. \& Rogers, T. L. (2010). Personal identification using the frontal sinus. J Forensic Sci, 55(3): 584-589.

Bleka, Ø., Wisløff, T., Dahlberg, P. S., Rolseth, V. \& Egeland, T. (2019). Advancing estimation of chronological age by utilizing available evidence based on two radiographical methods. Int J Legal Med, 133(1): 217-229.

Brasil. (1966) Lei no 5.081, de 24 de agosto de 1966. Regulamenta o exercício da odontologia no Brasil. Brasília: Diário Oficial da União; 1966

Brasil. (2005) Conselho Federal de Odontologia. Resolução nº 63, de 30 de junho de 2005. Consolidação das normas para procedimentos nos conselhos de odontologia. 
Bonavilla, J. D., Bush, M. A., Bush, P. J. \& Pantera, E. A. (2008). Identification of incinerated root canal filling materials after exposure to high heat incineration. J Forensic Sci, 53(2): 412-418.

Caldas, I. M., Magalhaes, T. \& Afonso, A. (2007). Establishing identity using cheiloscopy and palatoscopy. Forensic Sci Int, 165(1): 1-9.

Cameriere R., Ferrante L. \& Cingolani M. (2006). Age estimation in children by measurement of open apices in teeth. Int J Legal Med, 120(1): 49-52.

Cameriere, R., Ferrante, L., Molleson, T. \& Brown, B. (2008). Frontal sinus accuracy in identification as measured by false positives in kin groups. J Forensic Sci ,53(6): 1280-1282.

Cappella, A., Gibelli, D., Cellina, M., Mazzarelli, D., Oliva, A. G., De Angelis, D., ... \& Cattaneo, C. (2019). Three-dimensional analysis of sphenoid sinus uniqueness for assessing personal identification: a novel method based on 3D-3D superimposition. Int J Legal Med, $133(6)$ : $1895-1901$.

Chidambaram, R. (2016). Forensic Odontology: A Boon to Community in Medico-legal Affairs. JNMA J Nepal Med Asso, 54(201): 46-54.

Choi, I.G.; Duailibi-Neto, E.F.; Beaini, T.L.; da Silva, R.L. \&; Chilvarquer, I. (2018). The Frontal Sinus Cavity Exhibits Sexual Dimorphism in 3D Cone-beam CT Images and can be Used for Sex Determination. J Forensic Sci, 63(3): 692-698.

Christensen, A. M. (2005). Assessing the variation in individual frontal sinus outlines. AJPA: The Official Publication of the American Association of Physical Anthropologists, 127(3): 291-295.

David, M. P. \& Saxena, R. (2010). Use of frontal sinus and nasal septum patterns as an aid in personal identification: A digital radiographic pilot study. J Forensic Dent Sci, 2(2): 77.

Demirjian, A., Goldstein, H. \& Tanner, J. M. (1973). A new system of dental age assessment. Hum Biol, 45(2):211-227.

Eliášová, H. \& Dostálová, T. (2017). 3D Multislice and Cone-beam Computed Tomography Systems for Dental Identification. Prague medical report, 118(1): 14-25.

Flood, S. J., Franklin, D., Turlach, B. A. \& McGeachie, J. (2013). A comparison of Demirjian's four dental development methods for forensic age estimation in South Australian sub-adults. J Forensic Leg Med, 20(7): 875-883.

Freeman, A. J., Senn, D. R. \& Arendt, D. M. (2005). Seven hundred seventy eight bite marks: analysis by anatomic location, victim and biter demographics, type of crime, and legal disposition. J Forensic Sci, 50(6): JFS2005178-178.

Forrest, A. S. \& Wu, H. Y. H. (2010). Endodontic imaging as an aid to forensic personal identification. Aust Endod J, 36(2): 87-94.

Fonseca, G. M. \& Cantín, M. (2014). Lip print identification: People v. Davis or the convenient citation. J Forensic Leg Med. 25:6-7.

Fonseca, G. M., Ortíz-Contreras, J., Ramírez-Lagos, C. \& López-Lázaro, S. (2019). Lip print identification: current perspectives. J Forensic Leg Med, 65: 3238.

Gaêta-Araujo, H., Oliveira-Santos, N., Nascimento, E. H. L., Nogueira-Reis, F., Oenning, A. C., Groppo, F. C. \& Oliveira-Santos, C. (2021). A new model of classification of third molars development and its correlation with chronological age in a Brazilian subpopulation. Int J Legal Med, $135(2)$ : 639-648.

Gaikwad, R., Kamble, S., Rana, R., Jain, S., Gondivkar, S. \& Bajad, P. (2019). Rugae patterns as an adjunct to sex differentiation in forensic identification. Stomatologija, 21(3): 79-82.

Gadicherla, P., Saini, D. \& Bhaskar, M. (2017). Palatal rugae pattern: An aid for sex identification. J Forensic Dent. Sci., 9(1): 48.

Gibelli, D., De Angelis, D., Pucciarelli, V., Riboli, F., Ferrario, V. F., Dolci, C., ... \& Cattaneo, C. (2018). Application of 3D models of palatal rugae to personal identification: hints at identification from 3D-3D superimposition techniques. Int J Legal Med, 132(4): 1241-1245.

Gibelli, D., Cellina, M., Cappella, A., Gibelli, S., Panzeri, M. M., Oliva, A. G., ... \& Sforza, C. (2019). An innovative 3D-3D superimposition for assessing anatomical uniqueness of frontal sinuses through segmentation on CT scans. Int J Legal Med, 133(4): 1159-1165.

Goyal, M., Acharya, A. B., Sattur, A. P., \& Naikmasur, V. G. (2013). Are frontal sinuses useful indicators of sex?. J Forensic Leg Med, 20(2), 91-94.

Gruber, J., \& Kameyama, M. M. (2001). The role of radiology in forensic dentistry. Pesquisa Odontológica Brasileira, 15(3): 263-268.

Hamed, S. S., El-Badrawy, A. M., \& Fattah, S. A. (2014). Gender identification from frontal sinus using multi-detector computed tomography. J Forensic Radiol Imaging, 2(3): 117-120.

Herrera, L. M., Fernandes, C. M. S. \& Serra, M. C. (2013) Human identification by means of conventional and digital Cheiloscopy: a study of the literature. Rev Gaúcha Odontol., 61(1): 113-120.

Herrera, L. M., Fernandes, C. M. S. \& Serra, M. C. (2018) Evaluation of Lip Prints on Different Supports Using a Batch Image Processing Algorithm and Image Superimposition. J Forensic Sci, 63(1):122-129.

Interpol. (2018) Disaster Victim Identification Guide. https://www.interpol.int/How-we-work/Forensics/Disaster-Victim-Identification-DVI

Jain, A. \& Chowdhary, R. (2014). Palatal rugae and their role in forensic odontology. J Investig Clin Dent, 5(3): 171-178.

Jayaraman J., Roberts G.J., King N.M., Wong H.M. (2012). Dental age assessment of southern Chinese using the United Kingdom Caucasian reference dataset. Forensic Sci Int, 216(1-3): 68-72.

Kapoor, P. \& Chowdhry, A. (2018). Salivary signature in forensic profiling: A scoping review. J Forensic Dent Sci, $10(3)$ : 123. 
Kaul, R., Padmashree, S. M., Shilpa, P. S., Sultana, N., \& Bhat, S. (2015). Cheiloscopic patterns in Indian population and their efficacy in sex determination: A randomized cross-sectional study. J Forensic Dent. Sci, 7(2): 101.

Kaur, M., Singh, H., Dhillon, J. S., Batra, M. \& Saini, M. (2017). MTA versus Biodentine: review of literature with a comparative analysis. J Clin Diagn Res, $J C D R, 11(8): \mathrm{ZG} 01$.

Kessler, H. P. \& Pembie III, C. W. (1993). Forensic dental identification of casualties during Operation Desert Storm. Military medicine, 158(6): 359-362.

Khare, P., Raj, V., Chandra, S. \& Agarwal, S. (2014). Quantitative and qualitative assessment of DNA extracted from saliva for its use in forensic identification. J Forensic Dent Sci, 6(2): 81.

Kim, D. I., Lee, U. Y., Park, S. O., Kwak, D. S. \& Han, S. H. (2013). Identification using frontal sinus by three-dimensional reconstruction from computed tomography. J Forensic Sci, 58(1): 5-12.

Krishnan, V., Sreela, L. S., Mathew, P., \& Prasad, T. S. (2019). Radiographic evaluation of remodeling of mandible in adult South Indian population: Implications in forensic science. J Forensic Dent Sci, 11(3): 137.

Khalid, K., Yousif, S., \& Satti, A. (2016). Discrimination potential of root canal treated tooth in forensic dentistry. J Forensic Odontostomatol, 34(1): 19-26.

Khorate, M. M., Dinkar, A. D., \& Ahmed, J. (2014). Accuracy of age estimation methods from orthopantomograph in forensic odontology: a comparative study. Forensic Sci Int, 234: 184-e1.

Kommalapati, R. K., Katuri, D., Kattappagari, K. K., Kantheti, L. P. C., Murakonda, R. B., Poosarla, C. S., ... \& Baddam, V. R. R. (2017). Systematic analysis of palatal rugae pattern for use in human identification between two different populations. Iran J Public Health, 46(5): 602.

Lopez, T. T., Arruda, C. P., Rocha, M., de Oliveira Rosin, A. S. A., Michel-Crosato, E. \& Biazevic, M. G. H. (2013). Estimating ages by third molars: stages of development in Brazilian young adults. J Forensic Leg Med, 20(5): 412-418.

Malekzadeh, A. R., Pakshir, H. R., Ajami, S. \& Pakshir, F. (2018). The application of palatal rugae for sex discrimination in forensic medicine in a selected Iranian population. Iran J Med Sci, 43(6): 612 .

Manganotti, A. B. M., Faria, N. C., Franzak, F. D. \& Amaral, M. A. (2021). Análise e classificação da rugosidade palatina em um grupo de jovens adultas brasileiras. Res Soc Dev, 10(1): e46810111743.

Matsuda, S., Yoshida, H., Ebata, K., Shimada, I., \& Yoshimura, H. (2020). Forensic odontology with digital technologies: A systematic review. J Forensic Leg Med, 74: 102004

Michel, J., Paganelli, A., Varoquaux, A., Piercecchi-Marti, M. D., Adalian, P., Leonetti, G. \& Dessi, P. (2015). Determination of Sex: Interest of Frontal Sinus 3 D Reconstructions. J Forensic Sci, 60(2): 269-273.

Mishra, S. K., Mahajan, H., Sakorikar, R., \& Jain, A. (2014). Role of prosthodontist in forensic odontology. A literature review. J Forensic Dent Sci, 6(3): 154-159.

Moreira RP, Freitas AZVM. (1999) Dicionário de Odontologia Legal. Rio de Janeiro: Guanabara Koogan.

Mustafa, A. G., Allouh, M. Z. \& Alshehab, R. M. (2015). Morphological changes in palatal rugae patterns following orthodontic treatment. J Forensic Leg Med, 31: 19-22.

Muruganandhan, J. \& Sivakumar, G. (2011). Practical aspects of DNA-based forensic studies in dentistry. J Forensic Dent Sci, $3(1): 38$.

Nambiar, P., Naidu, M. D. \& Subramaniam, K. (1999). Anatomical variability of the frontal sinuses and their application in forensic identification. Clinical Anatomy: The Official Journal of the American Association of Clinical Anatomists and the British Association of Clinical Anatomists, 12(1): 16-19.

Nikam, S. S., Gadgil, R. M., Bhoosreddy, A. R., Shah, K. R., \& Shirsekar, V. U. (2015). Personal identification in forensic science using uniqueness of radiographic image of frontal sinus. J Forensic Odontostomatol, 33(1): 1-7.

Nolla, C. M. (1952). The development of permanent teeth (Doctoral dissertation, University of Michigan).

Oliveira, R. N. D., Daruge, E., Galväo, L. C. C. \& Tumang, A. J. (1998). Contribuiçäo da odontologia legal para a identificaçäo post-mortem. Rev Bras Odontol, 55: 117-122.

Oliveira, F. T., Capelozza, A. L. Á., Lauris, J. R. P. \& de Bullen, I. R. F. R. (2012). Mineralization of mandibular third molars can estimate chronological age-Brazilian indices. Forensic Sci Int, 219(1-3): 147-150.

Page, M., Lain, R., Kemp, R., \& Taylor, J. (2018). Validation studies in forensic odontology-Part 1: Accuracy of radiographic matching. Science \& Justice, 58(3): $185-190$

Pateria, A. H. \& Thakkar, K. (2011). Palatal rugae a stable landmark-A comparison between pre and post orthodontic patients. Int J Dent Clin, 3(4): 9-12.

Patil, M. S., Patil, S. B. \& Acharya, A. B. (2008). Palatine rugae and their significance in clinical dentistry: a review of the literature. $J$ Am Dent Assoc, 139(11): 1471-1478.

Patil, N., Karjodkar, F. R., Sontakke, S., Sansare, K. \& Salvi, R. (2012). Uniqueness of radiographic patterns of the frontal sinus for personal identification. Imaging science in dentistry, 42(4): 213. 
Poojya, R., Shruthi, C. S., Rajashekar, V. M. \& Kaimal, A. (2015). Palatal rugae patterns in edentulous cases, are they a reliable forensic marker? Int J Biomed Sci, 11(3): 109 .

Prabhat, M., Rai, S., Kaur, M., Prabhat, K., Bhatnagar, P., \& Panjwani, S. (2016). Computed tomography based forensic gender determination by measuring the size and volume of the maxillary sinuses. J Forensic Dent Sci, 8(1): 40-46.

Pretty, I. A., \& Hall, R. C. (2002). Forensic dentistry and human bite marks: issues for doctors. Hospital medicine, 63(8): 476-482.

Raina, P., Kulkarni, N. \& Shah, R. (2019). A comparative study of sagittal dental relationship using digital method of bite mark evaluation. J Forensic Dent Sci, 11(3): 125-132.

Roh, B. Y., Lee, W. J., Seo, J. U., Lee, U. Y. \& Lee, S. S. (2018). Analysis of forensic odontological examinations at the National Forensic Service of Korea from 2011 to 2015. Legal Med, 32: 37-42.

Santoro, V., Lozito, P., De Donno, A. \& Introna, F. (2011). Experimental study of bite mark injuries by digital analysis. J Forensic Sci, 56(1): 224-228.

Santos, C. (1946). Ficha odonto-rugopalatina. Rev bras Odontol, 4: 36-42.

Santos, K. C., Fernandes, C. M. S. \& Serra, M. C. (2011) Evaluation of a digital methodology for human identification using palatal rugoscopy. Braz J Oral Sci. 10(3): 199-203.

Seguí, M. A., Feucht, M. M., Ponce, A. C. \& Pascual, F. A. V. (2000). Persistent lipsticks and their lip prints: new hidden evidence at the crime scene. Forensic Sci Int, 112(1): 41-47.

Serra, M. C., Herrera, L. M. \& Fernandes, C. M. S. (2012) Importância da correta confecção do prontuário odontológico para identificação humana: relato de caso. Rev Assoc Paul Cir Dent, 66(2): 100-104.

Sharma, P., Saxena, S. \& Rathod, V. (2009). Comparative reliability of cheiloscopy and palatoscopy in human identification. Indian J Dent Res, 20(4): 453457.

Sharma, P., Sharma, N., Wadhwan, V. \& Aggarwal, P. (2016). Can lip prints provide biologic evidence? J Forensic Dent Sci, 8(3): 175.

Sheasby, D. R. \& MacDonald, D. G. (2001). A forensic classification of distortion in human bite marks. Forensis Sci Int, 122(1): 75-78.

Shukla, D., Chowdhry, A., Bablani, D., Jain, P. \& Thapar, R. (2011). Establishing the reliability of palatal rugae pattern in individual identification (following orthodontic treatment). J Forensic Odontostomatol, 29(1): 20-29.

Silva, M. B. I., Fernandes, C. M. S. \& Serra, M. C. (2012). Identificação humana por meio das marcas da orelha: métodos convencional e digital: um estudo da literatura. Rev Paul Odontol, 33(1): 10-19.

Silva, A. V., Costa, A. L. P., da Silva, M. L. C. A., Oliveira, M. B., Paranhos, L. R., Franco, A., \& de Oliveira Musse, J. (2020). Estudo anatômico do padrão labial entre mães e filhas do Nordeste brasileiro. Res Soc Dev, 9(11): e94691110545.

Suzuki, K. \& Tsuchihashi, Y. (1970). New attempt of personal identification by means of lip print. J Indian Dent Assoc, 42(1): 8-9.

Taneva, E., Kusnoto, B. \& Evans, C. A. (2015). 3D scanning, imaging, and printing in orthodontics. (pp. 147-188) In: Issues in contemporary orthodontics.

Taneva, E., Evans, C., \& Viana, G. (2017). 3D evaluation of palatal rugae in identical twins. Case reports in dentistry, 2017.

Tatlisumak, E., Asirdizer, M., \& Yavuz, M. S. (2011). Usability of CT images of frontal sinus in forensic personal identification. Theory and imaging of CT imaging and analysis. In Tech, Croatia, 257-265.

Thermadam, T. P., Chatra, L. \& Ahsan, A. (2020). Cheiloscopy in gender determination: A study on 2112 individuals. J Family Med Prim Care, 9(3): 13861390 .

Tsai, L. C., Su, C. W., Lee, J. C. I., Lu, Y. S., Chen, H. C., Lin, Y. C., ... \& Hsieh, H. M. (2018). The detection and identification of saliva in forensic samples by RT-LAMP. Forensic Sci Med Pathol, 14(4): 469-477.

Tuceryan, M., Li, F., Blitzer, H. L., Parks, E. T. \& Platt, J. A. (2011). A framework for estimating probability of a match in forensic bite mark identification. $J$ Forensic Sci, 56: S83-S89.

Ulery, B. T., Hicklin, R. A., Buscaglia, J.,\& Roberts, M. A. (2011). Accuracy and reliability of forensic latent fingerprint decisions. Proc Natl Acad Sci, 108(19): 7733-7738

Urzel, V. \& Bruzek, J. (2013). Dental age assessment in children: a comparison of four methods in a recent French population. J Forensic Sci, 58(5): 13411347.

Uthman, A. T., Al-Rawi, N. H., Al-Naaimi, A. S., Tawfeeq, A. S. \& Suhail, E. H. (2010). Evaluation of frontal sinus and skull measurements using spiral CT scanning: an aid in unknown person identification. Forensic Sci Int, 197(1-3): 124-e1.

Van der Velden, A., Spiessens, M. \& Willems, G. (2006). Bite mark analysis and comparison using image perception technology. J Forensic Odontostomatol, 24(1): 14 .

Weeratna, J. B. (2014). Are they dermatological lesions, bottle top burns or bite mark injuries? J Forensic Odontostomatol, 32(1): 1-8. 
Research, Society and Development, v. 10, n. 3, e20310313200, 2021

(CC BY 4.0) | ISSN 2525-3409 | DOI: http://dx.doi.org/10.33448/rsd-v10i3.13200

Willems, G., Van Olmen, A., Spiessens, B. \& Carels, C. (2001). Dental age estimation in Belgian children: Demirjian's technique revisited. J Forensic Sci, 46(4): 893-895.

Wrobel, K., Doroz, R., Porwik, P. \& Bernas, M. (2018). Personal identification utilizing lip print furrow based patterns. A new approach. Pattern Recognition, 81: 585-600.

Yoshino M., Miyasaka S., Sato H. \&, Seta S. (1987). Classification system of frontal sinus patterns by radiography. Its application to identification of unknown skeletal remains. Forensic Sci Int, 34(4): 289-299.

Zimmermann, B. G., Park, N. J. \& Wong, D. T. (2007). Genomic targets in saliva. Ann N Y Acad Sci, 1098: 184-191. 